\title{
Complete Genome Resource of Bacillus velezensis J17-4, an Endophyte Isolated from Stem Tissues of Rice
}

\author{
Zurong Shi, ${ }^{1,2}$ Wanwen Hong, ${ }^{2}$ and Qingwei Wang ${ }^{3, \dagger}$ \\ ${ }^{1}$ School of Biological Engineering, HuaiNan Normal University, Huainan 232038, China \\ ${ }^{2}$ College of Agriculture and Biology, Zhongkai University of Agriculture and Engineering, \\ Guangzhou 510225, China \\ ${ }^{3}$ Guangdong Laboratory for Lingnan Modern Agriculture, Guangdong Province Key Laboratory of \\ Microbial Signals and Disease Control, Integrative Microbiology Research Centre, South China \\ Agricultural University, Guangzhou 510642, China
}

\section{Abstract}

Dickeya zeae is the causative agent of rice root rot disease and causes severe harvest and economic losses. In this study, the Bacillus velezensis strain J17-4 with significant antagonism against $D$. zeae was used to generate DNA for sequencing. After assembly, a high-quality complete genome comprising only a circular chromosome was available. The genome sequence consists of a total of 3,877 prediction coding sequences and nine types of gene clusters involved in secondary metabolite production. This genome data will provide information for understanding the underlying mechanism of strain J17-4 antagonist against $D$. zeae and a new useful source for comparative genomics studies between strains isolated from various habitats.

\section{Genome Announcement}

The extensive use of chemical pesticides causes the accumulation of residual chemical compounds in the environment, which further results in environmental pollution and food safety problems. Numerous strains of Bacillus velezensis have been reported to suppress the growth of microbial pathogens; therefore, this species is attracting attention as a valuable biocontrol agent to circumvent the current undesirable effects. $B$. velezensis is an aerobic, Gram-positive, plant-associated bacterium which encodes $10 \%$ of its genome to the synthesis of antimicrobial molecules (Chen et al. 2007; Chowdhury et al. 2015). These antimicrobial compounds, including surfactin, iturins, bacillomycin-D, fengycins, bacillibactin, bacilysin, macrolactin, bacillaene, and difficidin, synthesized by $B$. velezensis display high antibacterial activity against several pathogenic microbes (Rabbee and Baek 2020). Apart from producing bioactive secondary metabolites against pathogenic microbes, $B$. velezensis was also found to contribute to plant protection by triggering induced systemic resistance in plants (Li et al. 2017), a process that activated the defense-associated genes of jasmonic acid and salicylic acid expression. With the development of sequencing technologies, genome-based approaches have been widely used to obtain the genomic and metabolic characteristics of Bacillus spp. (Chun et al. 2019). The first $B$. velezensis genome sequence was performed in 2007 (Chen et al. 2007) and, thus far, more than 150 whole-genome sequences of $B$. velezensis have become available in GenBank. These $B$. velezensis strains were isolated from different geographical locations and diverse environments, most of which were obtained from the plant rhizosphere or soil.

${ }^{\dagger}$ Corresponding author: Q. Wang; wangqingwei265@163.com

Z. R. Shi and W. W. Hong contributed equally.

The author(s) declare no conflict of interest.

Accepted for publication 8 July 2021.

๑) 2022 The American Phytopathological Society

\section{Funding}

This study was supported by Youth Innovative Talents Project (2016kqncx077) and Guangdong Forestry Science and Technology Innovation Project (2020KJCX009), and Guangzhou Science and Technology Planning Project (202102080488).

\section{Keywords}

biocontrol agent, cultural and biological practices, endophyte, field crops, genome, prokaryotes 
Table 1. Genomic attributes of endophytic biocontrol Bacillus velezensis strains

\begin{tabular}{lcc} 
Features & B. velezensis J17-4 & B. velezensis CC09 \\
Isolation source & Oryza sativa stems & Cinnamomum camphora leaves \\
Genome size (bp) & $3,929,768$ & $4,167,153$ \\
DNA G+C (\%) & 46.5 & 46.1 \\
Total genes & 3,877 & 4,094 \\
Protein encoding genes & 3,661 & 3,928 \\
RNA genes & 129 & 102 \\
Ribosomal RNAs & 27 & 24 \\
Transfer RNAs & 86 & 73 \\
Small RNAs & 16 & 5 \\
Pseudogenes & 87 & 64 \\
Genomic islands & 2 & - \\
NCBI accession & CP060420 & CP015443 \\
\hline
\end{tabular}

The host range of Dickeya zeae is very wide, including monocotyledons and dicotyledons (Hu et al. 2018). As the causative agent of rice root rot disease, D. zeae strain EC1 can significantly inhibit the germination of the seeds of many rice varieties with inoculation of only a few bacterial cells (Hussain et al. 2008). Previous studies have shown that some bacteria (i.e., Pseudomonas fluorescens, $P$. parafulva, and $B$. velezensis) isolated from soil with antagonistic activities against $D$. zeae have good efficacy in laboratory conditions ( $\mathrm{Li}$ et al. 2020). Therefore, biocontrol might be a powerful alternative to the use of synthetic chemicals to control rice root rot disease. Recently, we began to screen endophyte bacteria from resistant host plants of $D$. zeae strain EC1 and, providentially, we isolated the J17-4 strain from healthy and sterilized stems of rice variety Guang jinlizhan. This bacterium strain could significantly inhibit the growth of $D$. zeae strain EC1 with the maximum inhibition zones of $21.3 \mathrm{~mm}$ via confront culture. Furthermore, the J17-4 strain could significantly reduce the inhibitory effect of $D$. zeae strain EC1 against rice seed germination under laboratory inoculation conditions, and this strain was further identified as $B$. velezensis via taxonomic analysis (unpublished data). Here, we describe the complete genome sequence of this strain.

Genomic DNA of J17-4 was extracted using HiPure Bacterial DNA Kits (Magen, Guangzhou, China) according to the manufacturer's instructions. The whole-genome sequencing was manipulated on single-molecule real-time (SMRT) cells via Pacific Biosciences Sequel (PacBio, Menlo Park, CA, U.S.A.) according to standard protocols. Genome sequencing was also performed on the Illumina Novaseq 6000 sequencer using paired-end technology (PE 150). Raw data attained from SMRT sequencing runs were filtered and, in total, 168,843 subreads with an average read length of 7,041.8 bp were selected for assembly. Then, these subreads were de novo assembled by using the diploid-aware long-read assembler FALCONUnzip of FALCON (version 0.3.0) (Chin et al. 2016). Raw data from the Illumina platform were filtered using FASTP (version 0.20.0) by removing reads under standard to correct the genome sequences of SMRT sequencing to improve the quality of its assembly and determine the final genome sequences via Pilon (version 1.23) (Chen et al. 2018; Walker et al. 2014). After the complete genome was assembled, BLAST analysis was performed to identify the species that showed similarity to each scaffold. The completeness of strain J17-4 genome assembly was evaluated using the benchmarking universal single-copy orthologs (BUSCO v3.0) with bacilli_odb10 as a reference, based on evolutionarily informed expectations of gene content from near-universal single-copy orthologs (Simão et al. 2015). The analysis showed 299 complete and single-copy BUSCOs (99.01\%) and one missing BUSCO $(0.33 \%)$, indicating that strain J17-4 was a genome with high completeness (99.34\% complete).

Protein-coding regions were predicted using Prokka (version1.11) or NCBI prokaryotic genome annotation pipeline (Seemann 2014; Tatusova et al. 2016). Noncoding RNAs such as ribosomal RNAs (rRNAs) were predicted using rRNAmmer (version 1.2) (Lagesen et al. 2007), while transfer RNAs (tRNA) and small RNAs were identified by tRNAscan (version 1.3.1) and cmscan (version 1.1.2) (Lowe and Chan 2016; Nawrocki and Eddy 2013), respectively. Genomic islands were predicted using IslandViewer (version 4) (Bertelli et al. 2017). Interspersed and tandem repeat elements were identified by RepeatMasker (version 4.0.5) and TRF (Benson 1999; Tarailo-Graovac and Chen 2009), respectively. Secretory protein was predicted using SignalP4.0 (Petersen et al. 2011). Diverse protein databases, including 
the NCBI nonredundant protein database, UniProt/Swiss-Prot, Kyoto Encyclopedia of Genes and Genomes, gene ontology, and cluster of orthologous groups of proteins were applied to annotate functional protein categories. The secondary metabolite gene clusters were predicted using antiSMASH (version 4.1.0) (Blin et al. 2017).

The genome of $B$. velezensis strain $\mathrm{J} 17-4$ contained only one chromosome with no plasmid. The chromosome of strain J17-4 was 3,929,768 bp in length with 3,877 coding sequences. The additional features of this genome are summered in Table 1. Nine types of gene clusters involved in secondary metabolite production were predicted by genome analysis using antiSMASH. Twelve gene clusters were predicted associated with secondary metabolite production, including one nonribosomal peptide (nrps), two terpene, one T3pks, two transatpks, two transatpks-nrps, one bacteriocin-nrps, one otherks, and one other.

In the present study, we provide the complete genome sequence of $B$. velezensis, a valuable biocontrol agent isolated from healthy and sterilized rice stems. The genome of strain $\mathrm{J17}-4$ represents a new, useful source for future research on comparative genomics studies among stains separated from various environments.

\section{Data Availability}

The pure culture of $B$. velezensis $\mathrm{J} 17-4$, sequenced in this study, is preserved in the Chinese Culture Collection Center (accession number M2020308), Wuhan, China. The whole genomic data obtained in this study are both available at GenBank under BioProject accession number PRJNA657983 and Bio Sample ID SAMN15854525. The genome accession number described in this article is CP060420.

\section{Literature Cited}

Benson, G. 1999. Tandem repeats finder: A program to analyze DNA sequences. Nucleic Acids Res. 27:573-580.

Bertelli, C., Laird, M. R., Williams, K. P., Simon Fraser University Research Computing Group, Lau, B. Y., Hoad, G., Winsor, G. L., and Brinkman, F. S. L. 2017. IslandViewer 4: Expanded prediction of genomic islands for largerscale datasets. Nucleic Acids Res. 45:W30-W35.

Blin, K., Wolf, T., Chevrette, M. G., Lu, X., Schwalen, C. J., Kautsar, S. A., Duran, H., Santos, E., Kim, H., Nave, M., Dickschat, J. S., Mitchell, D. A., Shelest, E., Breitling, R., Takano, E., Lee, S. Y., Weber, T., and Medema, M. H. 2017. antiSMASH 4.0-improvements in chemistry prediction and gene cluster boundary identification. Nucleic Acids Res. 45:W36-W41.

Chen, S., Zhou, Y., Chen, Y., and Gu, J. 2018. Fastp: An ultra-fast all-in-one FASTQ preprocessor. Bioinformatics 34:i884-i890.

Chen, X. H., Koumoutsi, A., Scholz, R., Eisenreich, A., Schneider, K., Heinemeyer, I., Morgenstern, B., Voss, B., Hess, W. R., Reva, O., Junge, H., Voigt, B., Jungblut, P. R., Vater, J., Süssmuth, R., Liesegang, H., Strittmatter, A., Gottschalk, G., and Borriss, R. 2007. Comparative analysis of the complete genome sequence of the plant growth-promoting bacterium Bacillus amyloliquefaciens FZB42. Nat. Biotechnol. 25:1007-1014.

Chin, C. S., Peluso, P., Sedlazeck, F. J., Nattestad, M., Concepcion, G. T., Clum, A., Dunn, C., O'Malley, R., Figueroa-Balderas, R., Morales-Cruz, A., Cramer, G. R., Delledonne, M., Luo, C., Ecker, J. R., Cantu, D., Rank, D. R., and Schatz, M. C. 2016. Phased diploid genome assembly with single molecule real-time sequencing. Nat. Methods 13:1050-1054.

Chowdhury, S. P., Hartmann, A., Gao, X. W., and Borriss, R. 2015. Biocontrol mechanism by root-associated Bacillus amyloliquefaciens FZB42-A review. Front. Microbiol. 6:780.

Chun, B. H., Kim, K. H., Jeong, S. E., and Jeon, C. O. 2019. Genomic and metabolic features of the Bacillus amyloliquefaciens group- $B$. amyloliquefaciens, $B$. velezensis, and B. siamensis- revealed by pan-genome analysis. Food Microbiol. 77:146-157.

Hu, M., Li, J., Chen, R., Li, W., Feng, L., Shi, L., Xue, Y., Feng, X., Zhang, L., and Zhou, J. 2018. Dickeya zeae strains isolated from rice, banana and clivia rot plants show great virulence differentials. BMC Microbiol. 18:136.
Hussain, M., Zhang, H., Xu, J., Liu, Q., Jiang, Z., and Zhang, L. 2008. The acylhomoserine lactone-type quorum-sensing system modulates cell motility and virulence of Erwinia chrysanthemi pv. zeae. J. Bacteriol. 190:1045-1053.

Lagesen, K., Hallin, P., Rødland, E. A., Stærfeldt, H., Rognes, T., and Ussery, D. W. 2007. RNAmmer: Consistent and rapid annotation of ribosomal RNA genes. Nucleic Acids Res. 35:3100-3108.

Li, C., Hu, W. C., Pan, B., Liu, Y., Yuan, S. F., Ding, Y. Y., Li, R., Zheng, X. Y., and Shen, Q. 2017. Rhizobacterium Bacillus amyloliquefaciens strain SQRT3-mediated induced systemic resistance controls bacterial wilt of tomato. Pedosphere 27:1135-1146.

Li, J., Hu, M., Xue, Y., Chen, X., Lu, G., Zhang, L., and Zhou, J. 2020. Screening, identification and efficacy evaluation of antagonistic bacteria for biocontrol of soft rot disease caused by Dickeya zeae. Microorganisms 8:697.

Lowe, T. M., and Chan, P. P. 2016. tRNAscan-SE on-line: Integrating search and context for analysis of transfer RNA genes. Nucleic Acids Res. 44:W54-W57.

Nawrocki, E. P., and Eddy, S. R. 2013. Infernal 1.1: 100-Fold faster RNA homology searches. Bioinformatics 29:2933-2935.

Petersen, T. N., Brunak, S., Von Heijne, G., and Nielsen, H. 2011. SignalP 4.0: Discriminating signal peptides from transmembrane regions. Nat. Methods 8:785-786.

Rabbee, M. F., and Baek, K.-H. 2020. Antimicrobial activities of lipopeptides and polyketides of Bacillus velezensis for agricultural applications. Molecules 25:4973.

Seemann, T. P. 2014. Rapid prokaryotic genome annotation. Bioinformatics 30 : 2068-2069.

Simão, F. A., Waterhouse, R. M., loannidis, P., Kriventseva, E. V., and Zdobnov, E. M. 2015. BUSCO: Assessing genome assembly and annotation completeness with single-copy orthologs. Bioinformatics 31:3210-3212.

Tarailo-Graovac, M., and Chen, N. 2009. Using RepeatMasker to identify repetitive elements in genomic sequences. Curr. Protoc. Bioinf. 25:4.10.1-4.10.14.

Tatusova, T., DiCuccio, M., Badretdin, A., Chetvernin, V., Nawrocki, E. P., Zaslavsky, L., Lomsadze, A., Pruitt, K. D., Borodovsky, M., and Ostell, J. 2016. NCBI prokaryotic genome annotation pipeline. Nucleic Acids Res. 44:6614-6624.

Walker, B. J., Abeel, T., Shea, T., Priest, M., Abouelliel, A., Sakthikumar, S., Cuomo, C. A., Zeng, Q., Wortman, J., Young, S. K., and Earl, A. M. 2014. Pilon: An integrated tool for comprehensive microbial variant detection and genome assembly improvement. PLoS One 9:e112963. 